\title{
Treatment of Hypertension in Adults Above the Age of 60
}

Treating hypertension is a day to day duty of any physician. But when it comes to treat hypertension in elderly patients, especially above the age of 60 , achieving the target BP becomes difficult. Because of multiple co-morbidities and use of concomitant different kind of medications, very often, a single antihypertensive agent often fails to achieve the goal. Joint National Committee (JNC) publishes the guideline for hypertension management for several years and the latest one in JNC 8. In that also, the difficulty of treating hypertension in elderly patients have been mentioned.

In this editorial, we intend to focus on the latest updates as well as the modified target BP for elderly patients. The American College of Physicians (ACP) and the American Academy of Family Physicians (AAFP) have published an evidence-based clinical practice guideline on the appropriate systolic blood pressure target for adults 60 years old and older with hypertension very recently ${ }^{1}$.

Hypertension, an elevation of systemic arterial blood pressure, is one of the most common chronic diseases in the United States. About 65 percent of adults in the U.S. over the age of 60 have hypertension, and the disease affects about 29 percent of all adults in the nation. We have no such national data in our country. $\mathrm{ACP}$ and AAFP recommend that physicians initiate treatment in adults aged 60 years old and older with persistent systolic blood pressure at or above 150 millimeters of mercury $(\mathrm{mm} \mathrm{Hg})$ to achieve a target systolic blood pressure of less than $150 \mathrm{~mm} \mathrm{Hg}$ to reduce the risk of mortality, stroke, and cardiac events. Most benefits of targeting of less than $150 \mathrm{~mm} \mathrm{Hg}$ apply to individuals regardless of whether or not they have diabetes. Appropriate management of hypertension reduces the risk for cardiovascular disease, renal disease, cerebrovascular disease, and death. However, determining the most appropriate BP targets, particularly for adults aged 60 years or older, has been controversial. Debate about the goal for systolic BP (SBP) among adults treated for hypertension has intensified, especially in light of recent recommendations. In addition, when selecting BP targets for adults aged 60 years or older, clinicians need to consider comorbid conditions that could affect treatment choice. Treatments for hypertension include lifestyle modifications, such as weight loss, dietary modification, and increased physical activity, and antihypertensive medications, which commonly include thiazide-type diuretics, angiotensin-converting enzyme inhibitors (ACEIs), angiotensin-receptor blockers (ARBs), calcium-channel blockers, and $\beta$-blockers.

JNC 8 also suggested in patients 60 years or over, start treatment in blood pressures $>150 \mathrm{~mm} \mathrm{Hg}$ systolic or $>90 \mathrm{~mm} \mathrm{Hg}$ diastolic and treat to under those thresholds ${ }^{2}$.

The ACP/AA FP guideline notes that some patients may have falsely elevated readings in clinical settings ("white coat hypertension"). Therefore, it is important for physicians to ensure that they are accurately measuring blood pressure before initiating or changing treatment for hypertension. The most accurate measurements come from multiple blood pressure measurements made over time. These may include multiple measurements in clinical settings or ambulatory or home-monitoring.

\section{The guideline emphasize on two recommendations:}

ACP and AAFP recommend that physicians consider initiating or intensifying drug therapy in adults aged 60 years old and older with a history of stroke or transient ischemic attack to achieve a target systolic blood pressure of less than $140 \mathrm{~mm} \mathrm{Hg}$ to reduce the risk of recurrent stroke. 
ACP and AAFP recommend that physicians consider initiating or intensifying pharmacological treatment in some adults aged 60 years old and older at high cardiovascular risk, based on individualized assessment, to achieve a target systolic blood pressure of less than $140 \mathrm{~mm} \mathrm{Hg}$ to reduce the risk of stroke or cardiac events.

Increased cardiovascular risk includes all people with known vascular disease and among others, is defined as most patients with diabetes, individuals with chronic kidney disease with estimated glomerular filtration rate (eGFR) $<45 \mathrm{~mL} / \mathrm{min} /$ per $1.73 \mathrm{~m} 2$, metabolic syndrome (abdominal obesity, hypertension, diabetes, and dyslipidemia), and older age.

Whatever the guidelines describe every patient has separate merit in elderly as because of their co morbid conditions. A physician should weigh the risk versus benefit in all case management of treating hypertensive patients.

(J Bangladesh Coll Phys Surg 2017; 35: 1-2)

\section{Prof. Quazi Tarikul Islam}

Professor of Medicine

Popular Medical College

\section{Referrences:}

1. Qaseem A, Wilt TJ, Ritch R, Humphrey LL et al. A clinical practice guideline from American College of Physicians and American Academy of Family Physicians; Ann Intern Med ,144(2):2017

2. JNC 8 Guidelines for the Management of Hypertension in Adults. Am Fam Physician. 2014 Oct 1;90(7):503-504 